\title{
Novel Training Methods Based ANN for the Consumed Energy Forecasting
}

\author{
Arwa ben farhat ${ }^{1}$, adnen cherif ${ }^{2}$ \\ ${ }^{1}$ Enicarthage, Tunisia \\ ${ }^{2}$ University of Tunis, El manar \\ arwabenfarhat@gmail.com,adnen2.cherif@yahoo.fr
}

\begin{abstract}
Artificial Neural Networks have demonstrated best effectiveness and excellent scheduling capabilities in realizing many purposes like recognition, clustering, classification, management and even prediction. For this reason, we have used RBF based Artificial NN for the dynamic forecasting of load and Photovoltaic production using many operations like forecasting, training and validation of the data accuracy. For the validation, the Mean Absolute Percent Error is calculated in function of the most three relevant input parameters, which are previous load and Photovoltaic production measurements, seasonability and temperature or solar radiation data. This work has used real-time measurements of load and Photovoltaic production for their comparison with the predicted load data using RBFNN algorithms for the calculation of MAE and MAPE, to deduce the performance of forecasting algorithms including the accuracy of the forecasted data. This research paper has treated 2 goals. The first is the short-term energy and Photovoltaic production forecasting including training operations. The 2nd goal is the calculation of Mean Absolute Error and Mean Absolute Percent Error via the comparison between the forecasted data and real-time measurements to evaluate the reliability of forecasted data and the performance of the forecasting algorithms. By this way, the dynamic prediction algorithms were implemented, the predicted data were compared to the same time-series measurements and forecasted energy MAPE was calculated.
\end{abstract}

Keywords: Dynamic prediction algorithms, RBFNN, ISO, ErrCor, MAPE.

\section{INTRODUCTION}

The use of smart meters is not significant in some countries in developing state due their installation cost $[1,2]$. That is why, this research paper has treated the consumed energy forecasting goal to enhance the reliability of forecasted energy and the dynamic forecasting algorithms robustness, by the implementation of the necessary algorithms responsible for the energy forecasting $[3][4][5][6][7]$. Many works have treated the implementation of the forecasting algorithms for energy prediction and $\mathrm{PV}$ generation forecasting [10][11][12][13]. Renato et al. [14][15] have used WSN to measure electrical energy. They tested their measurement approach in an environment of largescale smart meters. Oveis Abedinia et.al. [18] have presented a reliable methodology for the solar radiation forecasting based neural networks using temperature, longitude, latitude...they have selected the pertinent input parameters using wika tool and have tested their method with the calculation of MAE then RMSE Yang Zhang et.al., [19] have proposed a novel load and price forecasting method based neural network for multi-level structure smart grid environment. Arwa Ben Farhat et.al, [20] have enhanced RBFNN by proposed new efficient second order method that can be applied for load forecasting. The research paper has concentrated on prediction of real-time algorithms used RBF training methods based neuro-fuzzy system for the forecasting of load in function of the input parameters as well as, the evaluation of the forecasted load data accuracy by the test of the performance of the used algorithms via the MAPE calculation. The proposed dynamic prediction algorithms have compared the forecasted data to evaluate the reliability and the accuracy of the predicted data as well as the performance of implemented forecasting algorithms. This research paper has been divided into several sections: Part 2 follows the ANN model used for the energy forecasting. Section 3 describes used load forecasting techniques. The part 4 presents the obtained energy forecasting scenarios considering the actual works; the conclusion presents the main contribution of this research study. 


\section{THE CONCEPTUAL STUDY OF THE ANN}

\subsection{The ANN model}

The ANN have shown strong capabilities in multipurpose approaches like recognition, classification, clustering and prediction, there are the most used technology according to [21] [22] that proved the best results in the prediction compared to the newest methods such as linear, nonlinear and fuzzy logic. ANN architecture is composed generally of three layers linked together by the interconnected nodes that integrate the network as shown in figure 1 . The first layer is used to receive input data to enter them into the model, the second layer known by the hidden layer is composed of a number of output parameters that are transferred from the first layer to be combined and trained in the second layer, the $3 \mathrm{rd}$ layer presents the obtained output from the processing and the transfer of final values in the hidden layer.

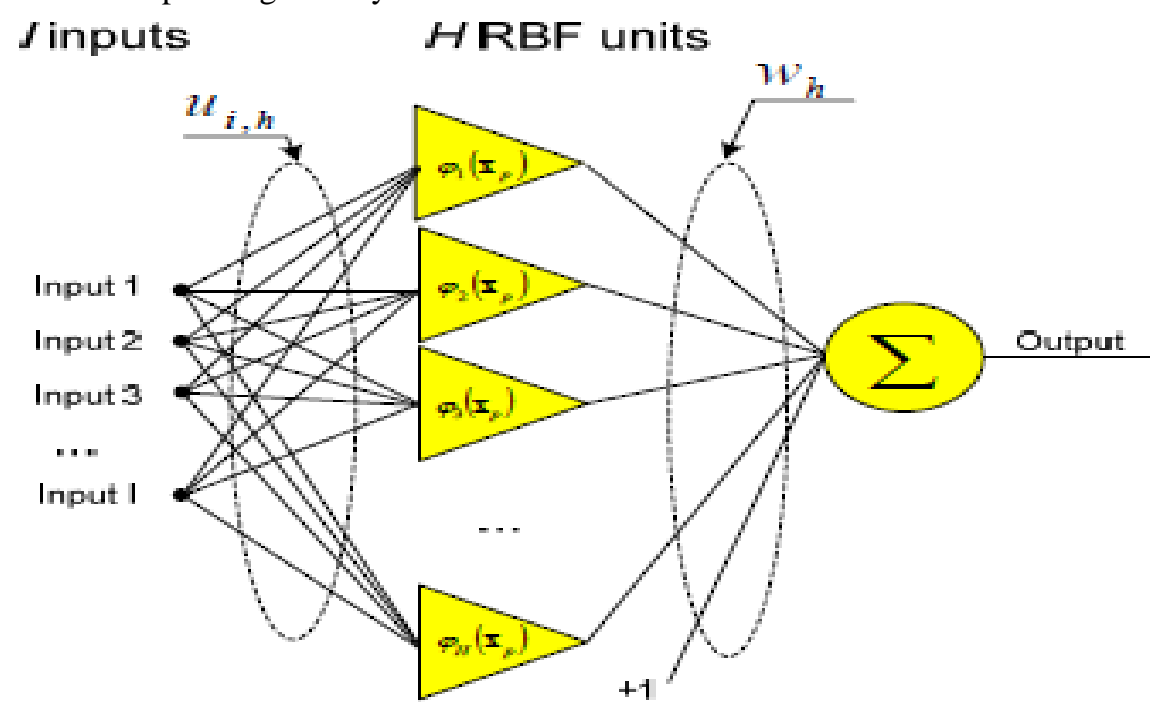

Figure 1. RBF based ANN model [21]

\section{THE PROPOSED APPROACHES}

\subsection{The used methods for the dynamic prediction approach}

There are several used machine learning methods based Radial Basis Function Artificial Neural Networks for the dynamic prediction of the consumed energy in the substations of smart grids environment, The most performant are Support Vector Regression method based classification and regression techniques which consisted on the fusion of groups of data according to the nearest Euclidian distance to build the support vectors based on the weight input and the locations of RBF using adjustment parameters inputs [21] .

We have also Extream Learning Machine based on the use of orthogonal least squares in order to detect the weights of output and the places of the centers of $\mathrm{RBF}[21]$.
The most performing and most reliable are Improved Second Order and Error Correction,

The Improved Second Order used iteration algorithms for the minimization of errors expressed in 1 st order by $\mathrm{Wk}+1=\mathrm{WK}-\mathrm{a} \mathrm{g}$ and in 2 nd order by the identity matrix. It consists on the adjustment of all parameters related to RBF [20].

The Error Correction is a RBFNN method witch uses a single analysis for the error validation [21].

Table 1 summarizes all the existing Radial basis Functions neural networks methods for the electrical energy forecasting with citing the advantages and the drawbacks of each used method.

\begin{tabular}{|c|l|l|l|}
\hline & Concept & Advantages & Drawbacks \\
\hline $\begin{array}{c}\text { Support } \\
\text { Vector } \\
\text { Regression } \\
\text { SVR }\end{array}$ & $\begin{array}{l}\text { Algorithms based on the fusion of groups of } \\
\text { data according to the nearest Euclidian } \\
\text { distance to build the support vectors based }\end{array}$ & high analysis time. & poor \\
results
\end{tabular}




\begin{tabular}{|c|c|c|c|}
\hline [21] & $\begin{array}{l}\text { on the weight input and the locations of } \\
\text { RBF using adjustment parameters inputs }\end{array}$ & & \\
\hline $\begin{array}{c}\text { Extream } \\
\text { Learning } \\
\text { Machine } \\
\text { ELM } \\
\text { [21] }\end{array}$ & $\begin{array}{l}\text { Algorithms based on the use of orthogonal } \\
\text { least squares in order to detect the weights } \\
\text { of output and the places of the centers of } \\
\text { RBF. }\end{array}$ & $\begin{array}{l}\text { Produced reasonable results } \\
\text { Faster than other algorithms } \\
\text { despite the use of RBF and SVR } \\
\text { inputs. }\end{array}$ & $\begin{array}{l}\text { the use of both RBF } \\
\text { and SVR inputs } \\
\text { complicate the } \\
\text { training. }\end{array}$ \\
\hline $\begin{array}{l}\text { Improved } \\
\text { Second } \\
\text { Order } \\
\text { ISO } \\
\text { [20] }\end{array}$ & $\begin{array}{l}\text { Iteration algorithms based on the } \\
\text { minimization of errors expressed in } 1 \mathrm{st} \\
\text { order by } \mathrm{Wk}+1=\mathrm{WK}-\mathrm{a} \mathrm{g} \text { and in } 2 \mathrm{nd} \text { order } \\
\text { by the identity matrix. } \\
\text { Adjustment of all parameters related to } \\
\text { RBF. }\end{array}$ & $\begin{array}{l}\text { Generates results with } 10 \text { times } \\
\text { more precision than SVR. }\end{array}$ & $\begin{array}{l}\text { Execution time is } \\
\text { very long. }\end{array}$ \\
\hline $\begin{array}{c}\text { Error } \\
\text { Correction } \\
\text { ErrCor } \\
{[21]}\end{array}$ & $\begin{array}{l}\text { characterized by a single analysis to obtain } \\
\text { the optimal solution. }\end{array}$ & $\begin{array}{l}\text { Error validation is more accurate } \\
\text { compared to ELM and SVR. } \\
\text { Error correction execution time } \\
\text { is faster compared to SVR and } \\
\text { ELM. }\end{array}$ & \\
\hline
\end{tabular}

Table.1. Summarizing table of the RBFNN methods for the data learning and performance test of the dynamic prediction algorithms

\subsection{The used energy forecasting method}

The used energy forecasting method has combined ISO and ErrCor methods ISO-ErrCor based on the trained RBF neuronal network for the load and PV generation forecasting in short and long term related to short-term measurements of the load and PV generation. The used energy forecasting method is taken from the prediction method of solar radiation [18], the latest method has based on artificial neuronal networks and the previous short and longterm weather information to forecast the solar radiations. It has used many parameters related to longitude, latitude, geographical features and temperature for its forecasting process.

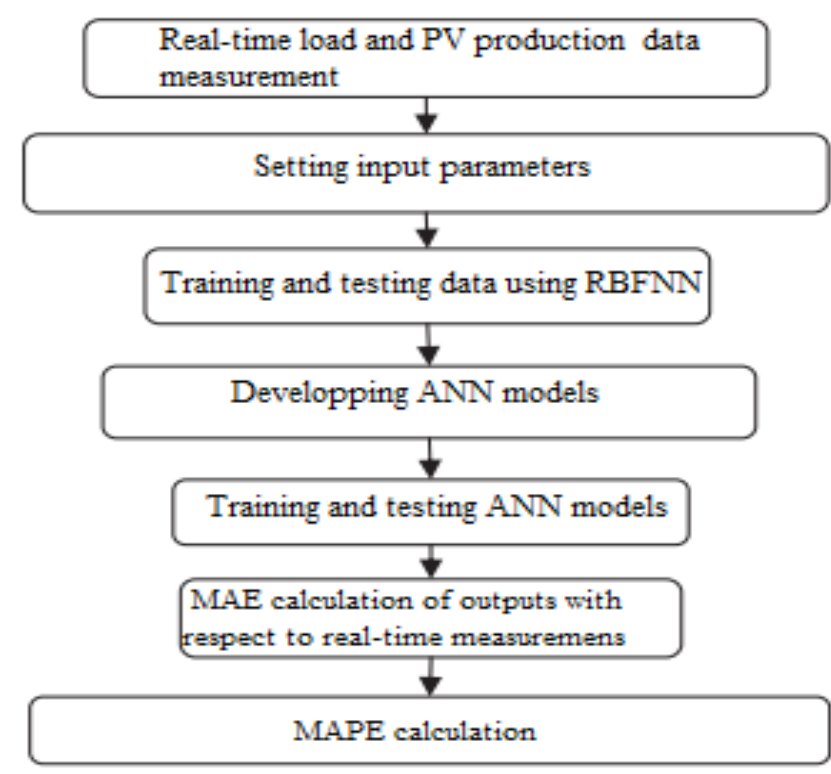

Figure.2. ANN dynamic prediction model 


\subsection{The used short-term energy forecasting algorithms}

The figure 3 presented the algorithms of energy forecasting used some input parameters (historical short-term load from electrical French company, the temperature and the day type. Figure. 3 presents energy-forecasting steps with training and validation of the obtained model based on RBF methods used ANN in machine learning. If the value of predicted energy is positive, then forecasting algorithm will be validated and the MAPE will be calculated, else the algorithm returned to the prediction process. The Load forecast is performed by pre-trained ANN or decision tree methods. These functions have used ANN or bagged trees to forecast energy in short and long-term.
Function $\mathrm{Y}=$ energy-Predict (input parameters)

$\mathrm{Y}=$ energy-Predict (model, Start to final date, hour, temperature, isWorkingDay))

This function shows the predictors' generation as a matrix forms.

Function [input parameters] $=$ genForecasters (historical-data, term, holiday)

$[\mathrm{X}$, dates, labels $]=$ genForecasters (historical-data, term, holiday)

The input parameters (data, term, holidays) are used to the generation of the predictor.

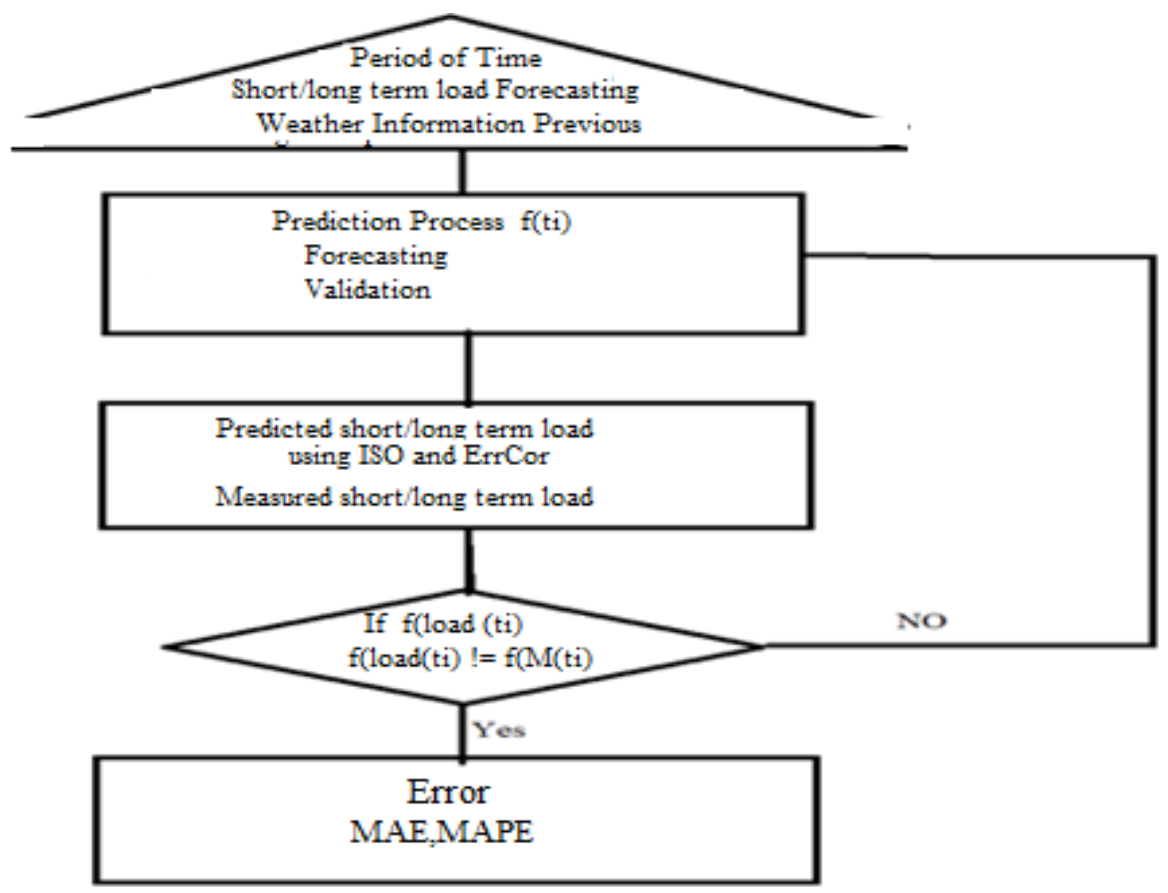

Figure.3. Energy use forecasting algorithm

\section{DISCUSSION OF THE RESULTS}

The energy forecasting techniques, use training and validation of the short or long term load and PV power production. The actual part will discuss the obtained outputs from the used energy forecasting methods.

\subsection{Energy forecasting results}

The used energy forecasting algorithms were used the historical load data available in «Ecomix website» database provided by the French electrical company and presented the scenarios of short-term historical load. The dynamic prediction codes were developed in Matlab, using the previous measurements, date type and weather information as input parameters to predict energy and power generation.

\subsubsection{The energy consumption forecasting demonstration}

Figure.4 follows the forecasted energy scenario, which presents variation at the level of energy consumption. The variation is related to electronic and domestic equipments over-use at 12 AM. The power drop at 5 PM. is related to the energy use 
reduction explained by period of time (break-time, holidays, weekdays...).

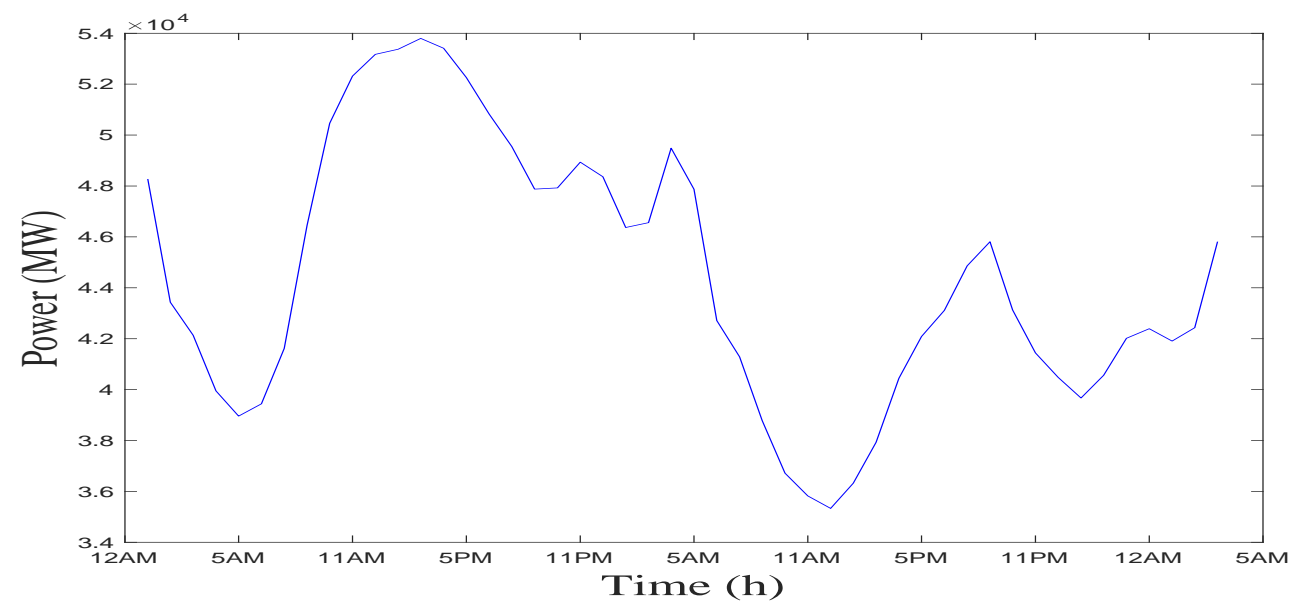

Figure 4. Day ahead load forecasting scenario

Figure.5 follows a week ahead energy forecasting scenario using RBFNN model and compares it to the real consumed power of the same time-series, we find that the obtained values of the forecasted energy are almost similar than the real power values of the same time-series, with $4 \%$ of MAPE and 2 s for the time training, which are low performance parameters compared to other studies using other forecasting techniques[13][21].

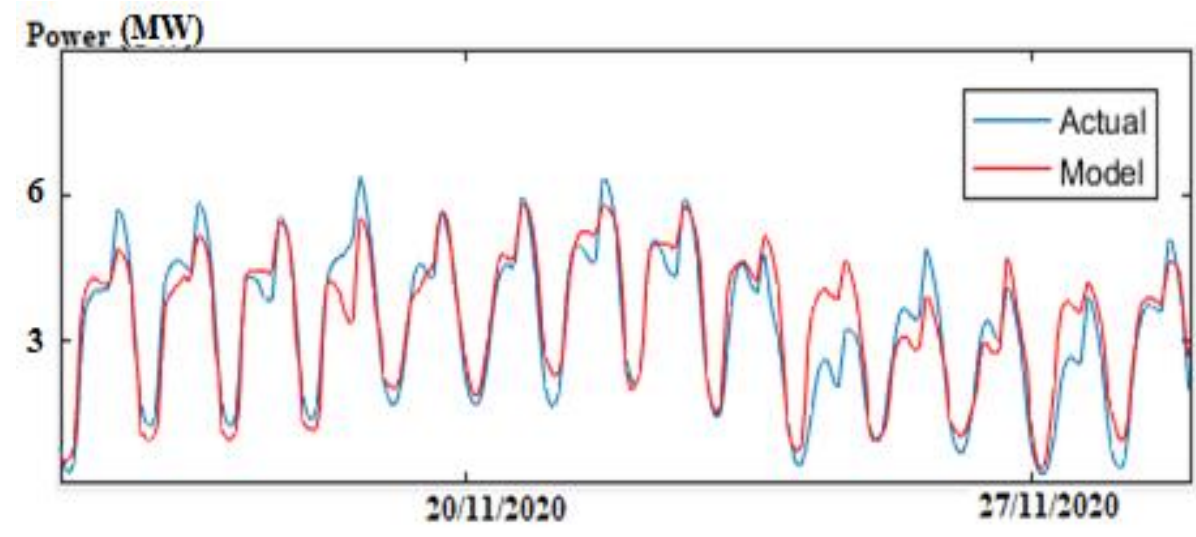

Figure 5. Week ahead power forecasting scenario compared to same time-series real power

\section{CONCLUSION}

This paper has summarized the used method based ANN based on RBF for the energy forecasting. The forecasting algorithms of the dynamic prediction method has presented a small mean absolute error percentage, demonstrated the predicted energy accuracy versus the measured energy of the same time series, which is almost similar, the used method present a fast time training during the error correction. This work has as contribution the reduction of forecasting error and time training. This research paper can be opened to other research perspectives such as demand side management.

\section{REFERENCES}

[1] Atilla Dönüket.al., "Metering and Data Processing in a Micro-Scale Area for Smart Grid Applications", IEEE International Istanbul Smart Grid Congress and Fair (ICSG), 2016.

[2] Abdul Khadar A, et.al. Research Advancements Towards in Existing Smart Metering over Smart Grid, International Journal of Advanced Computer Science and Applications, 2017

[3] A.S Kahweja et.al. Joint Bagged-Boosted ANN: Using ensemble Machine Learning to improve shortterm electricity Load Forecasting, Electric Power Systems Research, 2019. 
[4] Ravil Bikmetovet.al., Dynamic Prediction Capabilities of Smart Metering Infrastructure, IEEE North American Power Symposium (NAPS), 2015.

[5] E. Juan Zarate Perez, Performance Analysis of Bagging Feed-Forward Neural Network for Forecasting Building Energy Demand, Current Journal of Applied Science and Technology 2018.

[6] Samuel Atuahene, et.al. Artificial Neural Network based Artificial Intelligent Algorithms for Accurate Monthly Load Forecasting of Power Consumption, London Journal of Research in Science, Natural and Formal, Vol 19, Issue2, 2019 [7] Abandonner Reddy Salkuty, short-term electrical load forecasting using RBFNN considering weathers factors, Electrical Engineering, 2018.

[8] M. F. Khan, A. Jain, V. Arunachalam, and A. Pave than, «Communication technologies for smart metering infrastructure," presented at the 2014 IEEE Students' Conference, Bhopal, 2014.

[9] Haoyan Yang et.al., Short-term forecasting of Micro-grid based on grey correletion analysis and neural networ optimized by mind evolutionary algorithm, IEEE PES Innovative Smart Grid Technologies Asia, 2019.

[10] Ravil Bikmetov et.al. "Dynamic prediction capabilities of Smart Metering Infrastructure", Conference Paper, October 2015.

[11] Gabriel Trierweiler Ribeiro et.al., Enhanced ensemble structures using wavelet neural networks applied to shortterm load forecasting, Engineering Applications of Artificial Intelligence, 2020.

[12] Amit Kumar Yadav, Vikrant Sharma, Hasmat Malik, SS Chandel ,Daily array yeild of gridinteractive photovoltaic plant using relief attribute evaluator based Radial Basis Function Neural Network, Renewable and Sustainable Energy Reviews, 2017.
[13] Hamed H.H.Aly.,A Proposed Hybrid Load Forecasting Models of ANN,WNN and KF based on clustering Techniques for Smart Grids, Electric Power Systems Research, 2020..

[14]Peter Fecil'ak et.al. A non-intrusive Smart metering system: Analytics and simulation of power consumption, John Wiley \& Sons, 2019.

[15]Renato William R. de Souza et al. Deploying Wireless sensor networks-based smart grid for Smart meters monitoring and control, John Wiley \& Sons, 2015.

[16] Mohd. Salimuddin et.al. Smart Metering for Smart Power Consumption, International Journal for Research in Applied Science \& Engineering Technology, 2019.

[17] Krishna Paramatta Marimuthu et.al. Development and implementation of advanced metering infrastructure for efficient energy utilization in smart grid environment, John Wiley \& Sons, 2017.

[18] Oveis Abedinia et.al., Solar energy forecasting based on hybrid neural network and improved metaheuristic algorithm, Computational Intelligence, 2017.

[19] Yang Zhang et.al., A novel integrated price and load forecasting method in smart grid environment based on multi-level structure, 2019.

[20]Arwa ben Farhat, Cherif Adnen et.al, novel second order radial basis function neural network technique for enhanced load forecasting of PV power system, IJCSNS, 2021

[21] Bodgan M., A Novel RBF Training Algorithm for Short-Term Electric Load Forecasting and Comparative Studies, IEEE Transactions on Indistrual Electronics, 2015. 